\title{
Size-Dependent Characteristics of Indium-Seeded Si Nanowire
} Growth

\author{
F. Iacopi ${ }^{\mathrm{a}, \mathrm{z}}$ O. Richard, ${ }^{\mathrm{a}}$ Y. Eichhammer,${ }^{\mathrm{a}, \mathrm{b}}$ H. Bender, ${ }^{\mathrm{a}}$ P. M. Vereecken, ${ }^{\mathrm{a}, *}$ \\ S. De Gendt, ${ }^{\mathrm{a}, \mathrm{c}, *}$ and M. Heyns ${ }^{\mathrm{a}, \mathrm{b}}$ \\ ${ }^{a}$ IMEC, B-3001 Leuven, Belgium, 3001 \\ ${ }^{b}$ MTM Department and ${ }^{c}$ Chemistry Department, Katholieke Universiteit Leuven, Leuven, Belgium, 3001
}

Si nanowires were grown by a vapor-liquid-solid mechanism using indium catalyst particles with sizes between 10 and $200 \mathrm{~nm}$ Transmission electron microscopy analysis indicates that the growth morphology of the nanostructures changes drastically when the particle size is only a few tens of nanometers. The larger wires are monocrystalline, growing with $\langle 111\rangle$ orientation and limited tapering. The simultaneously grown smaller wires appear strongly tapered, due to an accumulation of amorphous $\mathrm{Si}$ on the sidewalls, and present a poor crystalline quality. A model based on the solubility of Si in the In-Si eutectic is proposed to account for the size-dependent change of morphology.

(c) 2008 The Electrochemical Society. [DOI: 10.1149/1.2945800] All rights reserved.

Manuscript submitted April 1, 2008; revised manuscript received May 14, 2008. Published July 16, 2008.

Semiconductor nanowires have received increasing attention recently thanks to the large range of prospective applications. ${ }^{1,2}$ In particular, nanowhiskers could find use in microelectronics as a channel material for vertical complementary metal oxide semiconductor (CMOS) devices, enabling the exploration of new architectures $^{3}$ and device concepts. ${ }^{4}$ However, the requirements for the feasibility of such an application are extremely stringent. First, the nanowire growth process should be compatible with Si technology, and as a consequence, the well-known use of $\mathrm{Au}$ as a growth metal catalyst ${ }^{5,6}$ is not a favored route. Second, the full control and reproducibility of wire characteristics such as location, size, orientation, and crystalline quality are also major requirements. We have previously shown that high-yield vapor-liquid-solid (VLS) growth of monocrystalline Si nanowires can be achieved using indium catalyst nanoparticles in combination with a plasma-enhanced chemical vapor deposition (PECVD) method. ${ }^{7}$ The use of indium instead of gold makes the growth process compatible with Si technology requirements, as In, unlike $\mathrm{Au}$, does not create midgap states in the $\mathrm{Si}$ bandgap and is a p-type impurity (acceptor) that can act as a dopant for $\mathrm{Si}^{8}{ }^{8}$ The location and size of the wires grown through VLS are determined by the catalyst's size and position, ${ }^{9}$ whereas control of the orientation and the crystalline quality of the nanostructures strictly relies on obtaining $\mathrm{Si}$ whiskers growing along the $\langle 111\rangle$ direction epitaxially onto $\mathrm{Si}(111)$ substrates. ${ }^{10}$ However, Wu et al. ${ }^{11}$ had already reported that Au-mediated VLS growth leads to a preferential growth direction gradually changing from $\langle 111\rangle$ to $\langle 112\rangle$ and $\langle 110\rangle$ for small diameter Si nanowires (a few tens of nanometers). This phenomenon is explained by surface energy arguments, i.e., the preferential nucleation of the lowest free-energy $\{111\}$ and $\{100\}$ faces, ${ }^{11}$ and has been confirmed by other groups. ${ }^{12}$ In view of the possible use of Si nanowires in very advanced CMOS technologies (beyond the $22 \mathrm{~nm}$ node $^{13}$ ), it is important to achieve a comprehensive understanding of the growth characteristics when scaling down the sizes of the nanowires toward diameters of a few tens of nanometers.

By growing nanowires simultaneously from a wide size range of In nanoparticles, we have systematically studied how the crystalline characteristics of the Si wires depend on the diameter, and we confirm that there are crucial aspects of VLS growth that are sizedependent.

Si nanowires were grown at $600^{\circ} \mathrm{C}$ onto $\mathrm{Si}(100)$ substrates with In nanoparticles by means of PECVD with low radio-frequency plasma power at $13.56 \mathrm{MHz}$. ${ }^{7} \mathrm{SiH}_{4}$ was used as the source gas with a partial pressure of 0.12 Torr, mixed with $\mathrm{Ar}$ and $\mathrm{H}_{2}$. The In particles were deposited by electrodeposition and exposed to an in situ

\footnotetext{
* Electrochemical Society Active Member

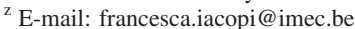

$\mathrm{H}_{2}$ plasma treatment prior to the growth process. ${ }^{7}$ This resulted in In particles with sizes ranging from $200 \mathrm{~nm}$ roughly down to $10 \mathrm{~nm}$, and with an initial contact angle on Si of about $115^{\circ} .{ }^{14}$

A systematic analysis of the grown wires by transmission electron microscopy (TEM) indicates that the larger wires are monocrystalline, with preferential growth orientation along the $\langle 111\rangle$ axis presenting $\{112\}$ and $\{110\}$ faces at the vapor-solid interface. A typical example is shown by the wire grown from a $200 \mathrm{~nm} \mathrm{In}$ particle in Fig. 1A. Such wires show limited tapering over about a $2.5 \mu \mathrm{m}$ wire length, except for an extended base at the very bottom of the structure. The same low-resolution TEM image in Fig. 1 also shows an example of wires grown from particles with a few tens of nanometers diameter $(10 \mathrm{~nm}$ in this case), indicating a very different morphology. Although both wires show comparable lengths, the wire grown from the $10 \mathrm{~nm}$ particle appears curved and has a much more pronounced tip-to-bottom tapering, clearly illustrated with higher magnification in Fig. 1B. A closer look at the base region of this wire (Fig. 1C) shows that the wire is composed of a crystalline

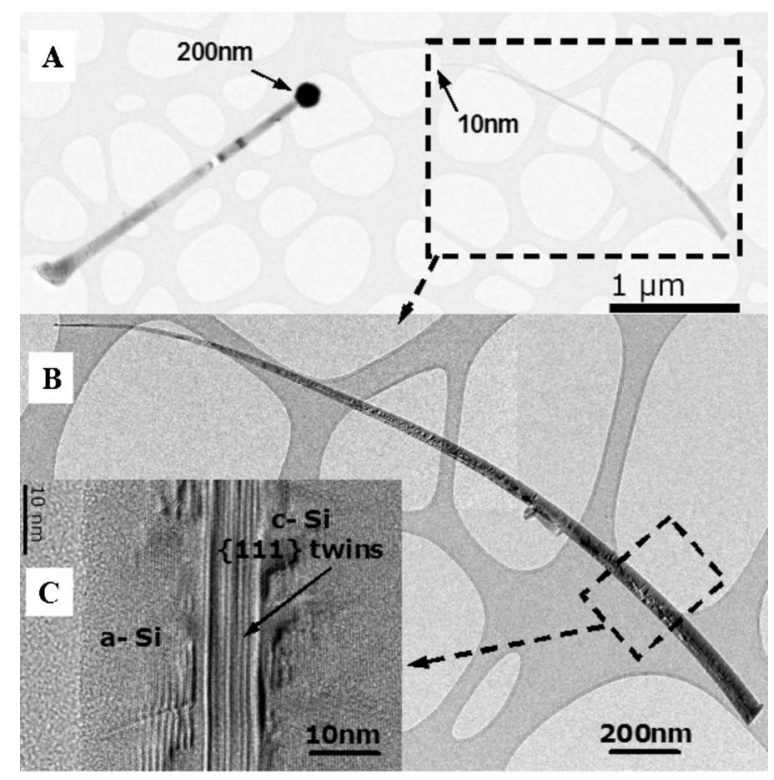

Figure 1. (A) Low-resolution TEM micrograph showing two wires simultaneously grown from a $200 \mathrm{~nm}$ diameter In particle and from a particle with diameter around $10 \mathrm{~nm}$. The wire grown from the small In particle shows strong tapering (higher magnification in B), and it is composed by a crystalline core with lamellar $\{111\}$ twinning parallel to the wire axis, surrounded by amorphous silicon (C). 


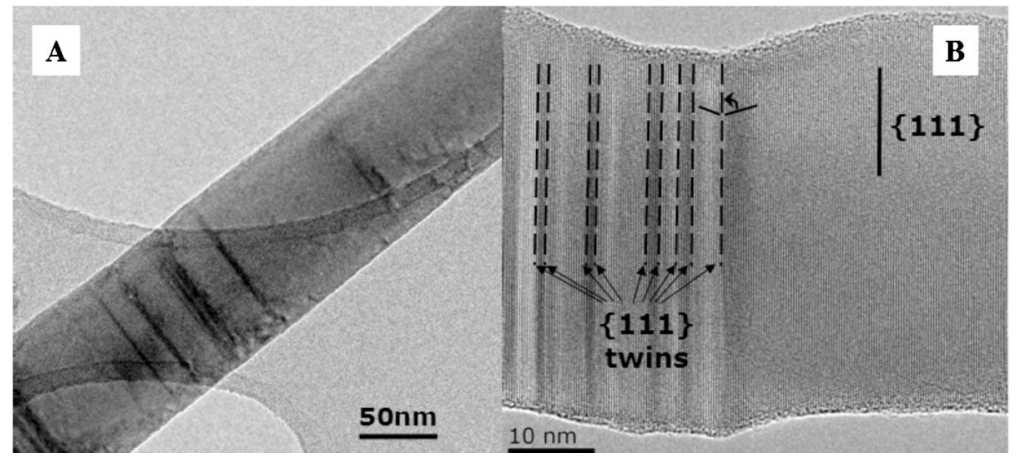

Figure 2. TEM micrographs from large diameter wires: (A) shows the region close to the base of a wire grown from a particle with about $200 \mathrm{~nm}$ diameter. (B) A higher resolution micrograph illustrates $\{111\}$ twinning perpendicular to the $\langle 111\rangle$ growth orientation, typical of the larger diameter wires. This wire was grown from a catalyst with about $100 \mathrm{~nm}$ diameter.

core with an almost constant diameter which is surrounded by a tapered amorphous Si wrap. Note that the core diameter is close to that of the catalyst particle. While it was not possible to deduce the exact growth orientation of the core crystal, lamellar $\{111\}$ twins are easily observed running parallel to the growth direction, all along the wire length. A recent work by Davidson et al. ${ }^{15}$ reports that this type of lamellar twins is generally associated with a $\langle 112\rangle$ growth direction, equally for IV and III-V semiconductor nanowires. A systematic TEM analysis of wires on the same sample indicates that the latter morphology tends to prevail as the particle/wire size decreases. The crystalline core of the smaller nanowires appears either monocrystalline with lamellar twins as in Fig. 1C or polycrystalline.

All wires grown from particles with size above $50 \mathrm{~nm}$ are found monocrystalline, and the wires grown from particles larger than 90$100 \mathrm{~nm}$ show an amorphous coating only a couple of nanometers thick, uniform along their sidewalls.

Figure $2 \mathrm{~A}$ shows a region close to the base of a wire grown from a particle of about $200 \mathrm{~nm}$ diameter. Whereas none of the analyzed large diameter wires showed $\{111\}$ twinning parallel to the growth direction, they often possess $\{111\}$ twins perpendicular to the $\langle 111\rangle$ growth direction, as shown with higher magnification in Fig. 2B. The angle between twins measured in Fig. $2 \mathrm{~B}$ is approximately $70^{\circ}$, which corresponds to the $70^{\circ} 32^{\prime}$ angle between two $\{111\}$ planes. ${ }^{16}$ According to Davidson et al., ${ }^{15}$ the nucleation of such a $\{111\}$ twin is favored by low nucleation energies (on the order of the thermal energy under growth conditions), and by a large contact angle between the catalyst particle and the whisker. When such a twinning occurs, the facets (either $\{112\}$ or $\{110\}$ in our case) restructure to a low-energy $\{111\}$ face. Through this facet restructuring, the angle for growth becomes higher than $90^{\circ}$ (straight nanowire walls), and the growth can only continue if the new facet can still be wetted by the catalyst. As the contact angle between the In particle and the Si wire is high, roughly around $115^{\circ}$, this type of twinning must be very common in In-seeded Si nanowire growth. Such perpendicular twinning is frequently found in Au-seeded III-V compound nanowires, and its nucleation was expected to be mostly suppressed for nonpolar materials as $\mathrm{Si}^{17}$ Indeed, this type of twinning is seldom observed for $\mathrm{Au}$-seeded $\mathrm{Si}$ nanowire growth, on which most reports are based, because of the smaller contact angle between the $\mathrm{Au}$ catalysts and the Si wire as compared to the In-Si system. It is likely that growth directions other than $\langle 111\rangle$ would suppress the perpendicular twinning; ${ }^{17}$ this could explain why we do not find perpendicular twins in the wires grown from the smaller In particles.

We analyzed so far the different types of $\{111\}$ twinning occurring in large vs small diameter wires, but we still need to elucidate the cause of the accumulation of amorphous Si leading to the tapering and for the polycrystallinity of the small wires. Both effects can be explained by the limited solubility of $\mathrm{Si}$ in the eutectic In-Si. First, the rate of lateral epitaxial $\mathrm{Si}$ growth directly from the gas phase, without the mediation of the catalyst particle, is expected to be rather limited and defective in chemical vapor deposition environments at temperatures below $680-700^{\circ} \mathrm{C}$. ${ }^{18}$ This means that most dissociated $\mathrm{Si}$ species impinging on the substrate or directly onto the wire would rather diffuse up to the In particle to precipitate into crystalline form from the liquid phase. ${ }^{19}$ The surface diffusion of the $\mathrm{Si}$ adatoms is not the limiting factor in our growth conditions, as the larger wires have comparable lengths but do not show an accumulation of amorphous $\mathrm{Si}$ at their base. However, the solubility of Si into the In particle is limited and the total amount of soluble Si atoms scales with the particle volume $V_{\mathrm{p}}$, whereas the direct impingement rate of $\mathrm{Si}$ on the wire sidewalls is proportional to the wire lateral area $A_{\mathrm{w}}$. When the amount of $\mathrm{Si}$ species supplied from direct impingement onto the wire exceeds the solubility/oversaturation limit of $\mathrm{Si}$ in the particle, $\mathrm{Si}$ is going to accumulate onto the wire sidewalls, mostly as amorphous material. For a rough trend estimation of this effect, we can make two approximations: we assume that the increase in the lateral wire area by possible tapering is negligible, and that the contact angle $\theta$ of the particle with the Si wire does not change with particle size. With $r$ the particle radius, the wire radius is assumed constant and can be expressed as $r \sin \theta$ as we have $\theta>90^{\circ}$. We can estimate the trend of the ratio $\Psi$ of the amounts of Si impinging directly on the lateral area of the wire with length $L$ to the amount of Si that can be dissolved into the particle as

$$
\Psi \propto \frac{A_{\mathrm{w}}}{V_{\mathrm{p}}}=\frac{3 \times 2 \pi r \sin \theta L}{4 \pi r^{3}}=\frac{3}{2} \frac{\sin \theta L}{r^{2}}
$$

Equation 1 can also be alternatively read in terms of the fraction $(1 / \Psi)$ of silicon species supplied by direct impingement that can actually dissolve into the particle, which scales as the square of the particle radius $r$, and as the inverse of the wire length $L$. As long as the total amount of $\mathrm{Si}$ species fed to the catalyst from the vapor phase and from the diffusion from the sidewalls of the wire stays below the saturation limit in the particle, the trend in Eq. 1 will not have any influence. However, the ratio $\Psi$ from Eq. 1 is expected to grow out of balance for small diameters. This means that, for a given wire length, there is a particle radius threshold linked to the $\mathrm{Si}$ supply rate from the gas phase and its oversaturation limit in the In-Si eutectic particle, below which the trend of Eq. 1 sidewall deposition of Si will become dominant.

Figure 3 shows the observed trend for wire tapering vs the particle radius $(r)$. The amount of tapering was calculated as the percentage ratio $\left(r_{2}-r_{1}\right) / r_{1}$, where $2 r_{1}$ is the diameter at the tip of the wires and $2 r_{2}$ is the diameter of the wires at a fixed chosen distance from the tip of $500 \mathrm{~nm}$ ( $L$; see the inset of Fig. 3). The measured amount of wire tapering for $r \geq 50 \mathrm{~nm}$ flattens out to a constant value around $10 \%$ and the corresponding excess lateral volume is completely crystalline. Hence, this limited amount of tapering can be attributed to lateral epitaxial growth directly from the vapor phase. However, for $r<50 \mathrm{~nm}$ the additional material deposited at the sidewalls becomes prevalently amorphous and the extent of tapering increases while decreasing the particle radius. Although the tapering trend vs $r$ in Fig. 3 cannot be put into direct comparison with Eq. 1, the tapering behavior is in agreement with the existence of a radius threshold below which the Si vapor species hitting the sidewalls will deposit at the impinging site, mostly as amorphous material. 


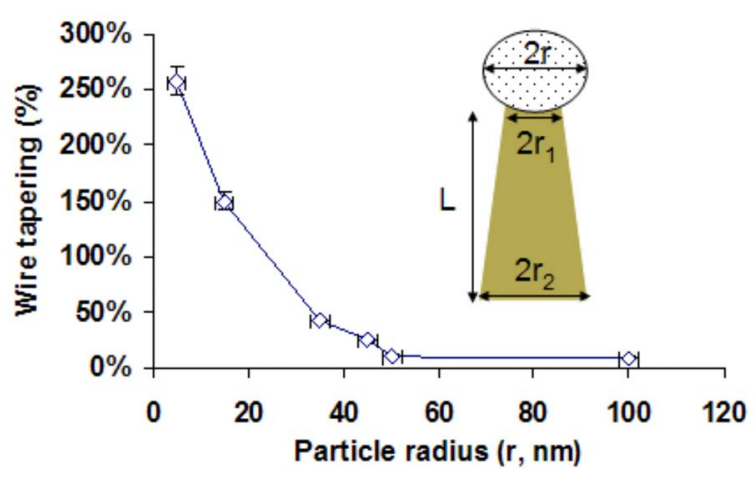

Figure 3. (Color online) Amount of tapering estimated from the TEM images of nanowires grown from different particle sizes at a fixed distance from the tip of $500 \mathrm{~nm}$. The extent of tapering increases when the particle radius decreases below $50 \mathrm{~nm}$, whereas above this radius limit the tapering trend flattens out at a constant value around $10 \%$.

The accumulation of $\mathrm{Si}$ on the wire sidewalls in an amorphous rather than crystalline form could also be associated with the appearance of $\{111\}$ facets as the wire diameter scales down. It is known that vapor phase $\mathrm{Si}$ epitaxy on $\mathrm{Si}(111)$ surfaces is more problematical. ${ }^{20}$ However, this phenomenon alone would neither account for the size-dependent tapering extent nor for the occurrence of polycrystallinity in the small wires.

The strong size-related limitation in the total amount of $\mathrm{Si}$ atoms soluble in the indium particles indicates that the supply of Si needs to be tuned differently according to the size range, as the optimal dilution needed for growing from small particles may be subcritical for initiating growth from the larger ones. Our studies indicated that $\mathrm{SiH}_{4}$ partial pressures above 0.05 Torr are needed to obtain growth from In particles with a diameter above $50 \mathrm{~nm}$ at $600^{\circ} \mathrm{C}$.

Furthermore, an estimation of the amount of $\mathrm{Si}$ dissolved in a eutectic In-Si alloy particle of $20 \mathrm{~nm}$ diameter, using a eutectic composition of 0.004 at $\% \mathrm{Si}^{21}$ and a liquid molar volume for In of $1.62 \times 10^{22}$ atoms $/ \mathrm{cm}^{3}, 22$ yields less than $10 \mathrm{Si}$ atoms. This means that the small liquid In particles are immediately driven into supersaturation with $\mathrm{Si}$, in such a way that the balance between the $\mathrm{Si}$ supply and the precipitation of crystalline Si will be subcritical and difficult to control. Discontinuous Si precipitation is most probably the reason why many of the small wires indicated a polycrystalline morphology.

In conclusion, the In catalyst-assisted growth of $\mathrm{Si}$ nanowires shows major size-dependent characteristics that have crucial implications for the controlled manufacturing of nanowires for advanced semiconductor technology nodes. While the larger wires are monocrystalline, oriented along the $\langle 111\rangle$ direction, and present $\{112\}$ or $\{110\}$ faces, the wires grown from particles with a few tens of nanometers diameter are either polycrystalline, or monocrystalline with $\{111\}$ twins extending throughout the wire length, indicative of a $\langle 112\rangle$ growth direction. Our work confirms the fact that small diameter wires tend to show different preferential growth orientation different from $\langle 111\rangle$. Furthermore, it is shown that the negligible amounts of $\mathrm{Si}$ that can be dissolved in very small eutectic In-Si particles limits the control of the crystalline quality of nanowires with a few tens of nanometers diameter, leading to polycrystallinity and pronounced tapering by the accumulation of amorphous Si. This limitation could be at least partially relieved by the use of catalyst materials with somehow higher eutectic Si solubilities. Because Au is not a favored candidate for microelectronic applications, the use of indium particles alloyed with other metals could be considered. The limitation concerning the change of preferential orientation for small wires is another crucial point, as the control on the wire orientation by epitaxial growth is lost. Possible solutions such as approaches based on a constrained growth into a predefined template ${ }^{23,24}$ need to be investigated further to ensure the feasibility of grown nanowires for microelectronic applications.

This work was partially supported by the EU programs NODE 015783 and SEA-NET integrated project IST-027982. We acknowledge Matty Caymax from IMEC for helpful discussions.

IMEC assisted in meeting the publication costs of this article.

\section{References}

1. Y. Huang, X. Duan, Y. Cui, L. J. Lauhon, K. H. Kim, and C. M. Lieber, Science, 294, 1313 (2001)

2. Z. Li, Y. Chen, X. Li, T. I. Kamins, K. Nauka, and R. S. Williams, Nano Lett., 4, 245 (2004).

3. C. Thelander, P. Agarwal, S. Brongersma, J. Eymery, L. F. Feiner, A. Forchel, M. Scheffler, W. Riess, B. J. Ohlsson, U. Gösele, and L. Samuelson, Mater. Today, 9, 28 (2006).

4. A. S. Verhulst, W. G. Vandenberghe, K. Maex, and G. Groeseneken, Appl. Phys. Lett., 91, 053102 (2007)

5. G. A. Bootsma and H. J. Gassen, J. Cryst. Growth, 10, 223 (1971).

6. S. Hofmann, C. Ducati, R. J. Neill, S. Piscanec, A. C. Ferrari, J. Geng, R. E. Dunin-Borkowski, and J. Robertson, J. Appl. Phys., 94, 6005 (2003)

7. F. Iacopi, P. M. Vereecken, M. Schaekers, M. Caymax, N. Moelans, B. Blanpain, O. Richard, C. Detavernier, and H. Griffiths, Nanotechnology, 18, 505307 (2007).

8. S. M. Sze, Physics of Semiconductor Devices, Wiley Interscience, New York (1981).

9. R. S. Wagner and W. C. Ellis, Appl. Phys. Lett., 4, 89 (1964).

10. A. P. Levitt, Whisker Technology, Chap. 2, p. 70, John Wiley \& Sons, New York (1970).

11. Y. Wu, Y. Cui, L. Huynh, C. J. Barrelet, D. C. Bell, and C. Lieber, Nano Lett., 4, 433 (2004)

12. P. Aella, Adv. Mater, 19, 2603 (2007)

13. International Technology Roadmap for Semiconductors, www.itrs.net (2007).

14. The contact angle was estimated from cross-sectional scanning electron micrographs at room temperature.

15. F. M. Davidson, D. C. Lee, D. D. Fanfair, and B. A. Korgel, J. Phys. Chem. C, 111, 2929 (2007).

16. E. I. Givarzizov, Oriented Crystallization on Amorphous Substrates, Chap. 1, p. 49, Plenum, New York (1998)

17. J. Johansson, L. S. Karlsson, C. P. T. Svensson, T. Martensson, B. A. Wacaser, K. Deppert, L. Samuelson, and W. Seifert, Nat. Mater, 5, 574 (2006).

18. H. M. Liaw and J. W. Rose, in Epitaxial Silicon Technology, Chap. 2, p. 75, J. Baliga, Editor, Academic Press, London (1986).

19. B. J. Baliga, in Epitaxial Silicon Technology, Chap. 3, J. Baliga, Editor, Academic Press, London, UK (1986).

20. D. J. Eaglesham, J. Appl. Phys., 77, 3597 (1995).

21. T. B. Massalski, Binary Alloy Phase Diagrams, 2nd ed., American Society for Metals, Metals Park, OH (1990).

22. G. Y. Shen, N. Sata, M. Newville, M. L. Rivers, and S. R. Sutton, Appl. Phys. Lett., 81, 1411 (2002).

23. T. Shimizu, T. Xie, J. Nishikawa, S. Shingubara, S. Senz, and U. Goesele, $A d v$. Mater. (Weinheim, Ger.), 19, 917 (2007).

24. T. F. Chung, L. B. Luo, Z. B. He, Y. H. Leung, I. Shafiq, Z. Q. Yao, and S. T. Lee, Appl. Phys. Lett., 91, 233112 (2007). 